\title{
Spectral Analysis of Information Density in Dialogue Predicts Collaborative Task Performance
}

\author{
Yang Xu and David Reitter \\ College of Information Sciences and Technology \\ The Pennsylvania State University \\ University Park, PA 16802, USA \\ yang.xulpsu.edu, reitterapsu.edu
}

\begin{abstract}
We propose a perspective on dialogue that focuses on relative information contributions of conversation partners as a key to successful communication. We predict the success of collaborative task in English and Danish corpora of task-oriented dialogue. Two features are extracted from the frequency domain representations of the lexical entropy series of each interlocutor, power spectrum overlap (PSO) and relative phase (RP). We find that PSO is a negative predictor of task success, while RP is a positive one. An SVM with these features significantly improved on previous task success prediction models. Our findings suggest that the strategic distribution of information density between interlocutors is relevant to task success.
\end{abstract}

\section{Introduction}

What factors affect whether information is conveyed effectively and reliably in conversations? Several theoretical frameworks have emerged that model dialogical behavior at different granularity levels. Can we use them to measure communicative effectiveness?

Grounding theory (Clark and Brennan, 1991) models a successful communication as a process during which "common ground" (i.e., mutual knowledge, beliefs etc.) is jointly built among interlocutors. The interactive alignment model (IAM) (Pickering and Garrod, 2004) proposes that the ultimate goal of dialogue is the alignment of interlocutors' situational model, which is helped by alignment at all other lower representation levels (e.g., lexical, syntactic etc.), driven by the psychologically well-documented priming effects.
Recently, empirical studies have verified the explanatory powers of the above-mentioned theories, especially the IAM, utilizing dialogues recorded and transcribed from various collaborative tasks conducted in laboratory settings (Reitter and Moore, 2007; Reitter and Moore, 2014; Fusaroli et al., 2012; Fusaroli and Tylén, 2016). In those studies, the quality of communication is directly reflected in the collaborative performance of interlocutors, i.e., how successful they are in accomplishing the task. Although they do not come to fully agree on which theoretical accounts of dialogue (e.g., interactive alignment vs. interpersonal synergy) provides better explanations (see Section 2.1 for details), the majority of these studies have confirmed that the alignment of certain linguistic markers, lexical items, or syntactic rules between interlocutors correlates with task success.

What is missing from the picture, however, is the computational understanding of how strategies of interaction and the mix of information contributions to the conversation facilitate successful communication. This is understandable because those higher level concepts do not directly map onto the atomic linguistic elements and thus are much more difficult to define and operationalize. In the present study, we intend to explore this missing part of work by characterizing how the interaction between interlocutors in terms of their information contributions affects the quality of communication.

\subsection{An information-based approach}

Recent work has already used information theory to study the dynamics of dialogue. Xu and Reitter (2016b) observed that the amount of lexical information (measured by entropy) from interlocutors of different roles, converges within the span of topic episodes in natural spoken dialogue. Anon (2017) interpret this converging pattern as a re- 
flection of the dynamic process in which the information contributed by two interlocutors fluctuates in a complementary way at the early stage, and gradually reaches an equilibrium status. $\mathrm{Xu}$ and Reitter (2016b) also correlated this entropy converging pattern with the topic shift phenomenon that frequently occurs in natural conversation $(\mathrm{Ng}$ and Bradac, 1993), and proposed that it reflects the process of interlocutors building the common ground that is necessary for the ongoing topics of conversation.

Based on Xu and Reitter's (2016) finding that entropy converging pattern repeatedly occurs within dialogue (though not necessarily at strictly regular intervals), it is reasonable to expect that after applying some spectral analysis techniques (time space to frequency space conversion) to the entropy series of dialogue, the frequency space representations should demonstrate some patterns that are distinct from white noise, because the periodicity properties in time space are captured.

Furthermore, we expect that how the frequency representations of two interlocutors correlate provides some information about the higher level properties of dialogue, e.g., the task performance etc. The thought is intuitive: If we imagine the entropy series from two interlocutors as two ideal sinusoidal signals $s_{1}$ and $s_{2}$ (supposedly of different frequencies, $f_{1}$ and $f_{2}$ ) (Figure 1), then the observed converging pattern can be thought of as a segment from the full spans of the signals. Then the frequency space properties, such as how close $f_{1}$ and $f_{2}$ are, and the phase difference $\phi$ between them, will definitely affect the shape of the converging pattern (solid lines in Figure 1). As Xu and Reitter (2016b) argues that the converging segment reflects the grounding process between interlocutors, it is reasonable to expect that the shape and length of this segment are reflective of how well interlocutors understand each other, and the overall collaborative performance as well.

Based on the above considerations, the goal of the present study is to explore how the frequency space representations of the entropy series of dialogue are correlated with the collaborative performance of task. We first demonstrate that entropy series satisfy the prerequisites of spectral analysis techniques in Section 4. Then we use two frequency space statistics, power spectrum overlap (PSO) and relative phase (RP), to predict task success. The reasons of using these two specific in-

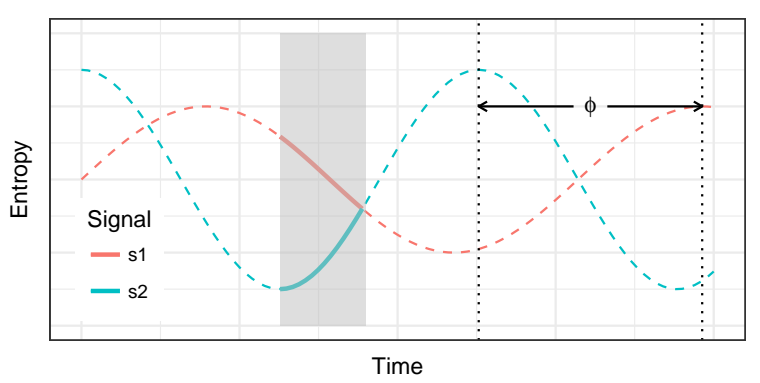

Figure 1: Analogizing the entropy converging patterns reported by $\mathrm{Xu}$ and Reitter (2016b) to a segment from two periodic signals. The shadowed area and the solid lines indicate the observed entropy convergence between interlocutors. The dashed lines are the imaginary parts of the ideal signals.

dices are discussed in Section 2.3, and their definitions are given in Section 3.3. The results are shown in Sections 5 to 7, and the implications are discussed.

\section{Related Work}

\subsection{The success of dialogue}

The interactive-alignment model (IAM) (Pickering and Garrod, 2004) stipulates that communication is successful to the extent that communicators "understand relevant aspects of the world in the same way as each other" (Garrod and Pickering, 2009). Qualitative and quantitative studies (Garrod and A. Anderson, 1987; Pickering and Garrod, 2006; Reitter and Moore, 2014) have revealed that the alignment of linguistic elements at different representation levels between interlocutors facilitates the success of task-oriented dialogues.

More recently, different theoretical accounts other than IAM, such as interpersonal synergy (Fusaroli et al., 2014) and complexity matching (Abney et al., 2014) have been proposed to explain the mechanism of successful dialogue from the perspective of dynamic systems. Fusaroli and Tylén (2016) compare the approaches of interactive alignment and interpersonal synergy in terms of how well they predict the collective performance in a joint task. They find that the synergy approach is a better predictor than the alignment approach. Abney et al. (2014) differentiate the concepts of behavior matching and complexity matching in dyadic interaction. They demonstrate the acoustic onset events in speech signals exhibit power law clustering across timescales, and the 
complexity matching in these power law functions is reflective of whether the conversation is affiliative or argumentative.

The perspective taken by the present study has some common places with Fusaroli and Tylén (2016) and Abney et al.'s (2014) work: we view dialogue as an interaction of two dynamic systems. The joint decision-making task used by Fusaroli and Tylén (2016) resulted in a small corpus of dialogue in Danish, which we will use for the present study.

\subsection{Information density in natural language}

Information Theory (Shannon, 1948) predicts that the optimal way to communicate is to send information at a constant rate, a.k.a. the principle of entropy rate constancy (ERC). The way humans use natural language to communicate also follows this principle: by computing the local per-word entropy of the sentence (which, under the prediction of ERC, will increase with sentence position), ERC is confirmed in both written text (Genzel and Charniak, 2002; Genzel and Charniak, 2003; Keller, 2004; Qian and Jaeger, 2011) and spoken dialogue (Xu and Reitter, 2016b; Xu and Reitter, 2016a). The theory of uniform information density (UID) extends ERC to syntactic representations (Jaeger, 2010) and beyond.

The information density in language, i.e., the distribution of entropy (predictability), reveal the discourse structure to some extent. For example, entropy drops at the boundaries between topics (Genzel and Charniak, 2003; Qian and Jaeger, 2011), and increases within a topic episode in dialogue (Xu and Reitter, 2016b) (see Section 1.1). The entropy of microblog text reflects changes in contextual information (e.g., an unexpected event in a sports game) (Doyle and Frank, 2015).

In sum, per-word entropy quantifies the amount of lexical information in natural language, and therefore fulfills the needs of modeling the information contribution from interlocutors.

\subsection{Spectral analysis methodology}

Spectral analysis, also referred to as frequency domain analysis, is a pervasively used technique in physics, engineering, economics and social sciences. The key idea of it is to decompose a complex signal in time space into simpler components in frequency space, using mathematical operations such as Fourier transform (Bracewell, 1986).
The application of spectral analysis in human language technology mainly focuses on processing the acoustic signals of human voice, and capturing the para-linguistics features relevant to certain tasks (Schuller et al., 2013). For example, Bitouk et al. (2010) find that utterance-level spectral features are useful for emotion recognition. Gregory Jr and Gallagher (2002) demonstrate that spectral information beneath $0.5 \mathrm{kHz}$ can predict US president election outcomes. However, we are not aware of the usage of spectral analysis in studying linguistic phenomena at higher representation levels than the acoustic level.

For our study, we are looking for some techniques that can capture the coupling between two signals at frequency space. The nature of the signal (whether it is language-related or not) should not be the first concern from the perspective of methodology. Therefore, studies outside the field of speech communication and linguistics could also be enlightening to our work.

After searching the literature, we find that the spectral analysis techniques that Oullier et al. (2002) and Oullier et al. (2008) use to study the physical and social functions of human body movement are useful to our research goal. In Oullier et al.'s (2002) work, subjects stood in a moving room and were to track a target attached to the wall. A frequency space statistics, power spectrum overlap (PSO), was used to demonstrate the coupling between motion of the room and motion of the subject's head. Stronger coupling effect (higher PSO) was found in the tracking task than a no-tracking baseline. PSO in nature quantifies how much the frequency space representations of two signals (power spectrum density) overlap. It allows us to explore the frequency space coupling of two interlocutors' entropy series in dialogue.

Similarly, Oullier et al. (2008) used the metrics of peak-to-peak relative phase (RP) and PSO to study the spontaneous synchrony in behavior that emerges between interactants as a result of information exchange. The signals to be analyzed were the flexion-extension movement of index fingers of two subjects sitting in front of each other. Both metrics showed different patterns when the participants see each other or not. RP, in their work, measures the magnitude of delay between two signals, and it corresponds to the notion of $\phi$ in Section 1.1. 


\section{Methods}

\subsection{Corpus data}

Two corpora are examined in this study: the HCRC Map Task Corpus (A. H. Anderson et al., 1991) and a smaller corpus in Danish from a joint decision-making study (Fusaroli et al., 2012), henceforth $D J D$.

Map Task contains a set of 128 dialogues between two subjects, who accomplished a cooperative task together. They were given two slightly different maps of imaginary landmarks. One of them plays as the instruction giver, who has routes marked on her map, and the other plays as the instruction follower, who does not have routes. The task for them is to reproduce the giver's route on the follower's map. The participants are free to speak, but they cannot see each other's map. The whole conversations were recorded, transcribed and properly annotated. The collaborative performance in the task is measured by the PATHDEV variable, which quantifies the deviation between the paths drawn by interlocutors. Larger values indicate poorer task performance.

DJD contains a set of 16 dialogues from native speakers of Danish (11,100 utterances and 56,600 words). In Fusaroli et al.'s (2012) original study the participants were to accomplish a series of visual perception task trials, by discussing the stimuli they saw and reaching a joint decision for each trial. The collaborative performance is measured by the CollectivePerformance variable, which is based on a psychometric function that measures the sensitivity of the dyad's joint decision to the actual contrast difference of the trial (Fusaroli et al., 2012). Higher value of this variable indicates better task performance.

The Switchboard Corpus (Godfrey et al., 1992) is used to train the language model for estimating the sentence entropy in Map Task. The Copenhagen Dependency Treebanks Corpus ${ }^{1}$ is used for the same purpose for DJD.

\subsection{Estimating information density in dialogue}

The information density of language is estimated at the sentence level, by computing the per-word entropy of each sentence using a trigram language model trained from a different corpus. We consider a sentence to be a sequence of words, $S=$

\footnotetext{
${ }^{1}$ http://mbkromann.github.io/ copenhagen-dependency-treebank/
}

$\left\{w_{1}, w_{2}, \ldots, w_{n}\right\}$, and the per-word entropy is estimated by:

$$
H\left(w_{1} \ldots w_{n}\right)=-\frac{1}{n} \sum_{w_{i} \in W} \log P\left(w_{i} \mid w_{1} \ldots w_{i-1}\right)
$$

where $P\left(w_{i} \mid w_{1} \ldots w_{i-1}\right)$ is estimated by a trigram model that is trained from an outside corpus. The SRILM software (Stolcke, 2002) is used to train the language model and to compute sentence entropy.

Dialogue is a sequence of utterances contributed by two interlocutors. For the $k$ th dialogue whose total utterance number is $N_{k}$, we mark it as $D_{k}=$ $\left\{u_{i}^{k} \mid i=1,2, \ldots, N_{k}\right\}$, in which $u_{i}^{k}$ is the $i$ th utterance. Map Task contains annotations of sentence structure in utterances, and one utterance could consist of several sentences that are syntactically independent. Thus we further split $D_{k}$ into a sequence of sentence, $D_{k}=\left\{s_{i}^{k} \mid i=\right.$ $\left.1,2, \ldots, N_{k}^{\prime}\right\}$, in which $N_{k}^{\prime}$ is number of sentences in $D_{k}$. Since DJD lacks the sentence annotations, we do not further split the utterance sequence, and simply treat an utterance as a complete sentence.

Given a sequence $\left\{s_{i}^{k}\right\}$ (Map Task), or $\left\{u_{i}^{k}\right\}$ (DJD), we calculate the per-word entropy for each item in the sequence:

$$
H_{k}=\left\{H\left(s_{i}^{k}\right) \text { or } H\left(u_{i}^{k}\right) \mid i=1,2, \ldots, N_{k}^{\prime}\left(\operatorname{or} N_{k}\right)\right\}
$$

where $H\left(s_{i}^{k}\right)$ or $H\left(u_{i}^{k}\right)$ is computed according to Equation 1.

Then we split the entropy series $H_{k}$ into two sub-series by the source of utterances (i.e., who speaks them), resulting in $H_{k}^{A}$ for interlocutor $A$, and $H_{k}^{B}$ for interlocutor $B$. For Map Task, the two interlocutors have distinct roles, instruction giver and follower. Thus the resulting two entropy series are $H_{k}^{g}$ and $H_{k}^{f}$. These per-interlocutor entropy series will be the input of our next-step spectral analysis.

\subsection{Computing power spectrum overlap and relative phase}

The time intervals between utterances (or sentences) vary, but since we care about the average information contribution within a complete semantic unit, we treat entropy series as regular time series. The time scale is not measured in seconds but in turns (or sentences).

For a given dialogue $D_{k}$, we apply the fast Fourier transform (FFT) on its two entropy se- 
ries $H_{k}^{A}$ and $H_{k}^{B}$, and obtain the power spectra (or, power spectral density plots) of them, $P_{k}^{A}$ and $P_{k}^{B}$. The power spectra are estimated with the $p e$ riodogram method provided by the open source $\mathrm{R}$ software. The $\mathrm{Y}$ axis of a power spectrum is the squared amplitude of signal (or power), and $\mathrm{X}$ axis ranges from 0 to $\pi / 2$ (we do not have sampling frequency, thus the $\mathrm{X}$ axis is in angular frequency but not in $\mathrm{Hz}$ ).

The power spectrum overlap, $\mathrm{PSO}_{k}$, is calculated by computing the common area under the curves of $P_{k}^{A}$ and $P_{k}^{B}$ is calculated, and normalizing by the total area of the two curves (see Figure 2). $\mathrm{PSO}_{k}$ ranges from 0 to 1 , and a larger value indicates higher similarity between $P_{k}^{A}$ and $P_{k}^{B}$.

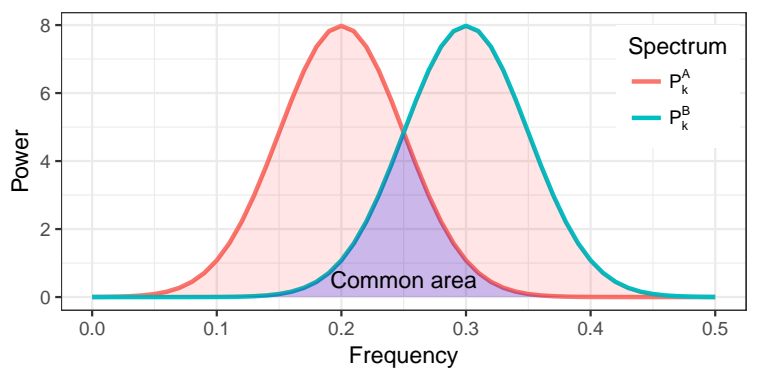

Figure 2: How PSO is computed. The blue shadow is the common area under two spectrums.

The relative phase (RP) between $H_{k}^{A}$ and $H_{k}^{B}$ is directly returned by the spectrum function in $R$. It is a vector of real numbers that range from 0 to $\pi$, and each element represent the phase difference between two signals at a particular frequency position of the spectrum.

\section{Prerequisites of Spectral Analysis}

Before proceeding to the actual analysis, we first examine whether the data we use satisfy some of the prerequisites of spectral analysis techniques. One common assumption of Fourier transforms is that the signals (time series) are stationary (Dwivedi and Subba Rao, 2011). Stationarity means that the mean, variance and other distributional properties do not change over time (Natrella, 2010). Another presumption we hold is that the entropy series contain some periodic patterns (see Section 1.1), which means their power spectrum should differ from that of white noise.

\subsection{Examine stationarity}

We use three pervasively used statistical tests to test the stationarity of our entropy series data: the
Table 1: Percentage stationary data

\begin{tabular}{lccc}
\hline Corpus & ADF & KPSS & PP \\
\hline Map Task & $82.4 \%$ & $95.5 \%$ & $100 \%$ \\
DJD & $100 \%$ & $81.3 \%$ & $100 \%$ \\
\hline
\end{tabular}

augmented Dickey-Fuller (ADF) test (Dickey and Fuller, 1979), the Kwiatkowski-Phillips-SchmidtShin (KPSS) test (Kwiatkowski et al., 1992), and the Phillips-Perron (PP) test (Phillips and Perron, 1988). The percentage of entropy series that pass the stationarity tests are shown in Table 1 . We can see that the majority of our data satisfy the assumption of stationarity, and thus it is valid to conduct Fourier transform on the entropy series.

The stationarity property seems contradictory to the previous findings about entropy increase in written text and spoken dialogue (Genzel and Charniak, 2002; Genzel and Charniak, 2003; Xu and Reitter, 2016b), because stationarity predicts that the mean entropy stays constant over time. We examine this in our data by fitting a simple linear model with entropy as the dependent, and sentence position as the independent variable, which yields significant (marginal) effects of the latter: For Map Task, $\beta=2.3 \times 10^{-3}, p<.05$, Adj- $R^{2}=$ $1.7 \times 10^{-4}$; For DJD, $\beta=7.2 \times 10^{-5}, p=$ $.06, A d j-R^{2}=2.2 \times 10^{-4}$. It indicates that the stationarity of entropy series does not conflict with the entropy increasing trend predicted by the principle of ERC (Shannon, 1948). We conjecture that stationarity satisfies because the effect size $\left(\operatorname{Adj-} R^{2}\right)$ of entropy increase is very small.

\subsection{Comparison with white noise}

Power spectra for all entropy series are obtained with an FFT. We compare them with those of white noise. The white noise data are simulated with i.i.d. random data points that are generated from normal distributions (same means and standard deviations as the actual data). Figure 3 shows the smoothed average spectrums of the actual entropy data and the simulated white noise data.

White noise signals should demonstrate a constant power spectral density (Narasimhan and Veena, 2005), and if the entropy series is not completely random, then their average spectrum should be flat. Linear models show that the average spectrums of the entropy data have slopes that are significantly larger than zero (For Map Task, 


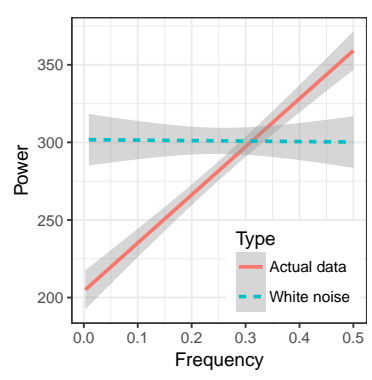

(a) Map Task

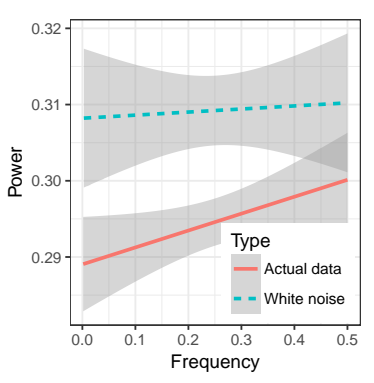

(b) DJD
Figure 3: Comparing the average power spectra of the actual entropy data and white noise. There are significant linear correlations between power (Y axis) and frequency (X axis) for the actual entropy data, which means the data are not completely random. Shadowed areas are $95 \%$ C.I.

$\beta=2.3 \times 10^{-2}, S E=9.4 \times 10^{-3}, p<.05$; for DJD, $\beta=314.1, S E=19.8, p<.001$ ), while the slopes of the white noise data are not significantly different from zero. This confirms our presumption that the entropy series of dialogue contains some periodic patterns that are identifiable in frequency space.

We also conduct Ljung-Box test (Ljung and Box, 1978) to examine how the entropy series is different from white noise. The null hypothesis is that the time series being tested is independent of the lagged sequence of itself. The test on a white noise series will give big $p$-values, for any lags greater than 0 , because of its randomness nature. We try several lags on each entropy series, and pick the smallest $p$-value. Consequently, we obtain a mean $p$-value of .23 on MapTask, and a mean $p$-value of .27 on DJD. Therefore, we cannot reject the null hypothesis for all the entropy series data, but the Type-I error of considering them as different form white noise is pretty low.

\section{PSO Predicts Task Success}

\subsection{Results of linear models}

We compute PSO for all the dialogues in Map Task and DJD and fit two linear models using PSO as predictor, with PATHDEV and CollectivePerformance as dependent variables respectively.

PSO is a reliable predictor in both models ( $p<$ $.05)$. The coefficients are shown in Table 2. Since PATHDEV is a measure of failure, but collaborative task performance is a measure of success, the negative correlation between PSO and collabora- tive task performance is consistent. Regression lines with residuals are plotted in Figure 4.

Table 2: Coefficients of PSO in predicting PATHDEV (Map Task) and CollectivePerformance (DJD). * indicates $p<.05$.

\begin{tabular}{lcccc}
\hline Dependent & $\beta$ & $S E$ & $F$ & Adj-R \\
\hline PATHDEV & 124.8 & 49.4 & $6.39^{*}$ & .045 \\
$\begin{array}{l}\text { Collective- } \\
\text { Performance }\end{array}$ & -40.9 & 15.9 & $6.60^{*}$ & .271 \\
\hline
\end{tabular}

Figure 4 (a) suggests a heteroscedasticity problem, because the right half of data points seem to stretch up along the $\mathrm{y}$ axis. This was confirmed by a Breush-Pagan test (Breusch and Pagan, 1979) $(B P=5.62, p<.05)$. To rectify this issue, we adopt a Box-Cox transformation (Box and Cox, 1964) on the dependent variable, PATHDEV, which is a typical way of handling heteroscedasticity. The new model that uses PSO to predict the Box-Cox transformed PATHDEV also yields significant coefficients: $\beta=3.85, S E=$ 1.67, $F(1,113)=5.32, p<.05$. Therefore, the correlation between PSO and PATHDEV is reliable.

As for DJD, due to the lack of data (we only have 16 dialogues), we do not run further diagnostics analysis on the regression model.

\subsection{Discussion}

The coupling of entropy series in frequency space is negatively correlated with task success. In other words, synchrony between interlocutors in terms of their information distribution hinders the success of collaboration. By "synchrony", we mean an overlap in the frequencies at which they choose to inject novel information into the conversation.

This conclusion seems contradictory to the perspective of interactive alignment at the first glance. However, here we are starting with a very highlevel model of dialogue that has does not refer to linguistic devices. Instead, we utilize the concept of "information density" and the entropy metric of natural language, to paint the picture of a system in which communicators inject novelty into the dialogue, and that each communicator does so regularly and with a set of overlapping frequencies. We assume that the rapid change of sentence entropy, i.e., the high frequency components in the spectrum, correspond to the moments in conversa- 


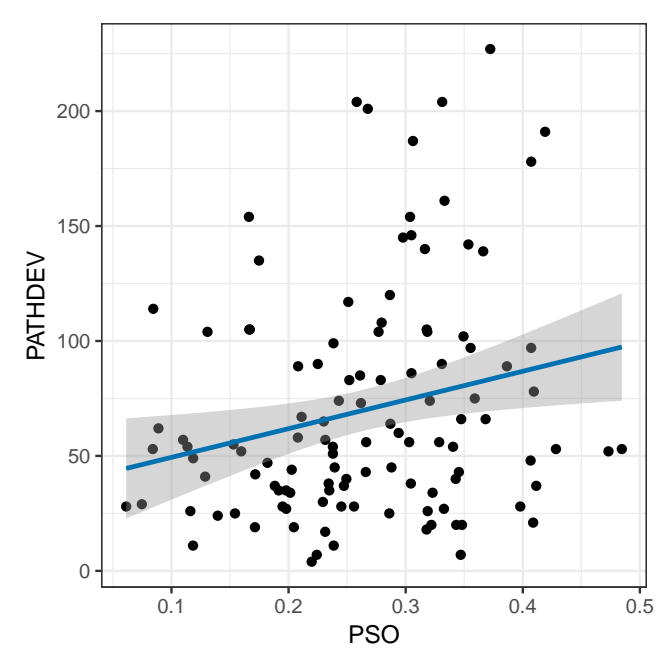

(a) Map Task

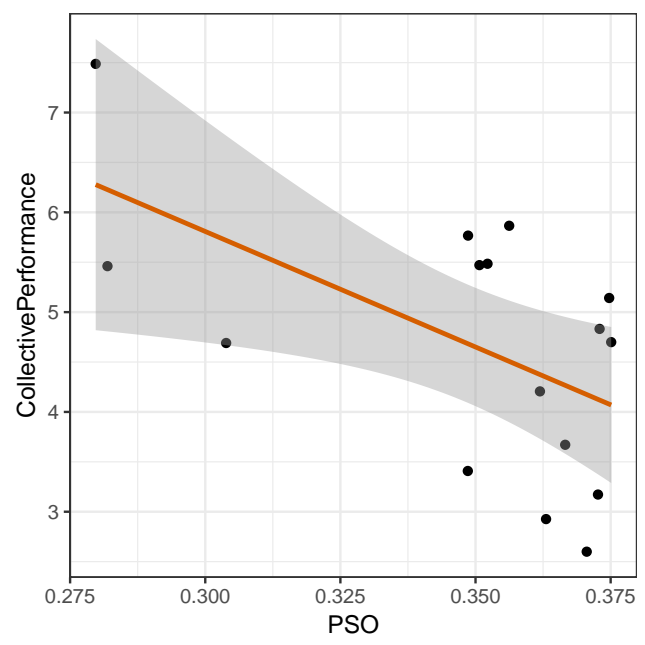

(b) DJD

Figure 4: Regression lines of linear models using PSO to predict PATHDEV in Map Task (a) and CollectivePerformance in DJD (b). Shadowed areas are 95\% C.I.

tion where one interlocutor brings relatively novel content to the table, such as a detailed instruction, a strange question, an unexpected response etc. This assumption is reasonable because previous work has shown that sudden change in entropy predicts topic change in dialogue (Genzel and Charniak, 2003; Qian and Jaeger, 2011; Xu and Reitter, 2016b).

We argue that higher synchrony (larger overlap in frequency space) in terms of how much novelty each interlocutor contributes, does not necessarily leads to better outcomes of communication. Rather, we would expect the correlation to be opposite (and our empirical results confirm this), because dialogue is a joint activity, in which a taking on different roles as interlocutors (e.g., the one who gives orders versus the one who follows) is often required to push the activity along (Clark, 1996). A dialogue with maximal synchrony or frequency overlap would be one where partners take turns at regular intervals. Perhaps because such regularity in turn-taking assigns no special roles to interlocutors, and because they engage in turntaking with no regard for content, it is not strange that such synchrony is disadvantageous.

Let's look at several scenarios of different synchrony levels between interlocutors: First, high synchrony due to both interlocutors contributing large amount of new information, which means there is more overlap near the high frequency band of spectrums. In this case, they are more likely to have difficulty in comprehending each other due to the potential information overload. Situations such as arguing, or both speakers asking a lot of questions are good examples. Second, high synchrony due to both interlocutors providing ineffective information, which indicates overlap in spectrums near the low frequency band. Obviously this type of ineffective communication is not helpful to the collaborative task. Third, low synchrony due to one interlocutor providing more information and the other one providing less, which means the overlap in spectrums is minimum. An example of this case is that one interlocutor is saying something important, while the other one is producing short utterances such "uh-huh", "yes", or short questions to make sure that they are on the same page, which is known as the back-channel mechanism in conversation (Oreström, 1983). This complementary style of communication allows them to build mutual understand of each other's intention, and thus reaches better collaborative performance.

\section{RP Predicts Task Success}

\subsection{Results of linear models}

We obtain the relative phase (RP) vector (absolute values) of all frequency components, and fit linear models using the mean of RP as predictor, and task performance as the dependent variable. We get non-significant coefficients for both models: For Map Task, $F(1,113)=.004, p>.05$; for DJD, $F(1,14)=.772, p>.05$. This suggests that the phase information of all frequency components in spectrum is not very indicative of task success. 
The power spectra describe the distribution of energy across the span of frequency components that compose the signal. The frequency components with higher energy (peaks in spectrum) are more dominant than those with lower energy (troughs) in determining the nature of the signal. Therefore it makes sense to only include the peak frequencies into the model, because they are more "representative" of the signal, and so the "noise" from the low energy frequencies are filtered out. Thus we obtain RP from the local peak frequency components, and use the mean, median, and maximum values of them as predictors. It turns out that for Map Task, the maximum of RP is a significant predictor (the mean and median are left out via stepwise analysis). For DJD, the mean of $\mathrm{RP}$ is a significant predictor of task success (when median and maximum are included in the model). (see Table 3).

Table 3: Coefficients of the linear models using the mean, median, and maximum values of RP from peak frequency components to predict task performance. $* p<.05, \dagger p<.1$.

\begin{tabular}{llccc}
\hline Corpus & Predictor & $\beta$ & $S E$ & $t$ score \\
\hline Map Task & $\max$ & -64.9 & 30.3 & $\mathbf{- 2 . 1 4}^{*}$ \\
\hline \multirow{3}{*}{ DJD } & mean & 15.6 & 5.7 & $\mathbf{2 . 7 6}^{*}$ \\
& median & -7.4 & 3.6 & $-2.06^{\dagger}$ \\
& $\max$ & -11.5 & 7.2 & -1.60 \\
\hline
\end{tabular}

From the significant effect of maximum RP in Map Task and mean RP in DJD, it is safe to state that RP is positively correlated with task performance. However, this relationship is not as straight-forward as PSO, because of the marginal effect at the opposite direction. A more finegrained analysis is required, but it is outside the scope of this study.

\subsection{Discussion}

The relative phase in frequency space can be understood as the "lag" between signals in time space. Imagine that we align the two entropy series from one dialogue onto the same time scale (just like Figure 1), the distance between the entropy "peaks" is proportionate to the relative phase in frequency space. Then, the positive correlation between relative phase and task performance suggests that relatively large delays between entropy
Table 4: $R^{2}$ performance on the HCRC MapTask task success prediction task (percentage of variance explained). 10-fold cross-validated by dialogue; same folds for each model. Reitter and Moore (2007) (R\&M) contained length and lexi$\mathrm{cal}$ and syntactic repetition features.

\begin{tabular}{ll}
\hline Model & $R^{2}$ \\
\hline R\&M & .17 \\
R\&M LENGTH only & .09 \\
R\&M LENGTH only $(\mathrm{C}=.5)$ & .1260 \\
R\&M (C=.5) & .1771 \\
R\&M + PSO + RP &. $\mathbf{2 8 2 6}$ \\
R\&M + PSO*RP & .2435 \\
R\&M LENGTH only + PSO*RP & .2494 \\
\hline
\end{tabular}

"surges" seen in each interlocutor are beneficial to collaborative performance.

The delay of entropy surges can be understood as a strategy for an interlocutor to distribute information in his or her own utterance accordingly with the information received. For example, after interlocutor $A$ contributes a big piece of information, the other one, $B$, does not rush to make new substantial contributions, but instead keeps her utterances at low entropy until it is the proper time to take a turn to contribute. This does not have to coincide with dialogic turn-taking.

This delay gives $B$ more time to "digest" the information provided by $A$, which could be an instruction that needs to be comprehended, or a question that needs to be thought about and so on. A relatively long delay guarantees enough time for interlocutors to reach mutual understanding. On the contrary, if $B$ rushes to speak a lot shortly after the $A$ 's input, then it will probably cause information overload and be harmful to communication.

Therefore, we believe that the RP statistic captures the extent to which interlocutors manage the proper "timing" of information contribution to maintain effective communication.

\section{Prediction Task}

Here we explore whether the frequency domain features, PSO and RP, can help with an existing framework that utilizes alignment features, such as the repetition of lexical and syntactic elements, to predict the success of dialogue in MapTask (Reitter and Moore, 2007). 
R\&M described an SVM model that takes into the repetition count of lexicons (LEXREP) and syntax structures (SYNREP), and the length of dialogues (LENGTH) as features. The full model achieves an $R^{2}$ score of .17 , which means that it can account for $17 \%$ of the variance of task success.

We add the new PSO and RP (mean, median and maximum RP features per dialogue are included) covariates to the original SVM model. An RBF kernel $(\gamma=5)$ was used. The cost parameter $C$ was (coarsely) tuned on different cross-validation folds to reduce overfitting on this relatively small dataset, and the R\&M's original full model was recalculated (shown in Table 4 as R\&M). Two models with PSO and RP interactions (once without the alignment/repetition features) are shown for comparison. (See Table 4).

Significant improvement in the model's explanatory power, i.e., $R^{2}$, is gained after the PSO and RP features are added. The best model we have is by adding PSO and RP as predictors without the interaction term (bold number in Table 4), which gives about $60 \%$ increase of $R^{2}$ compared to R\&M's full model. Note that even if we exclude the alignment features, and include only (LENGTH) and the frequency features (last row in Table 4), the performance also exceeds R\&M's full model.

The results indicate that the frequency domain features (PSO and RP) of the sentence information density can capture some hidden factors of task success that are unexplained by the alignment approach. It is not surprising that how people coordinate their information contribution matters a lot to the success of the collaboration. What we show here is that regular, repeated patterns of information-dense and information-sparse turns seem to make speakers more or less compatible with each other. Whether individuals have typical patterns (frequency distributions) of information density, or whether this is a result of dynamic interaction in each particular dialogue, remains to be seen.

\section{Conclusions}

The empirical results of the present study suggest that examining how the information contribution from interlocutors co-develops can provide a way to understand dialogue from a higher-level perspective, which has been missing in existing work.
Our work adds a brick to the series of endeavors on studying the linguistic and behavioral factors of successful dialogue, and for the first time (as far as we know) demonstrates quantitatively that the dynamics of not just "what" and "how" we say, but also "how much" we say and the "timing" of distributing what we say in dialogue, are relevant to the quality of communication. Although the way we model information in language is simply the entropy at lexical level, we believe the findings still reveal the nature of information production and processing in dialogue. We hope that by comparing and combining our methodology with other approaches of studying dialogue, we can reach a more comprehensive and holistic understanding of this common yet mysterious human practice.

\section{Acknowledgments}

We thank Riccardo Fusaroli for providing the DJD dataset. We have received very helpful input from Gesang Zeren in developing the initial ideas of this project. The work leading to this paper was funded by the National Science Foundation (IIS-1459300 and BCS-1457992).

\section{References}

Abney, D. H., Paxton, A., Dale, R., \& Kello, C. T. (2014). Complexity matching in dyadic conversation. Journal of Experimental Psychology: General, 143(6), 2304.

Anderson, A. H., Bader, M., Bard, E. G., Boyle, E., Doherty, G., Garrod, S., ... Miller, J. et al. (1991). The HCRC map task corpus. Language and Speech, 34(4), 351-366.

Bitouk, D., Verma, R., \& Nenkova, A. (2010). Class-level spectral features for emotion recognition. Speech Communication, 52(7), 613-625.

Box, G. E. \& Cox, D. R. (1964). An analysis of transformations. Journal of the Royal Statistical Society. Series B (Methodological), 211-252.

Bracewell, R. N. (1986). The Fourier transform and its applications. New York: McGrawHill.

Breusch, T. S. \& Pagan, A. R. (1979). A simple test for heteroscedasticity and random coefficient variation. Econometrica: Journal of the Econometric Society, 1287-1294.

Clark, H. H. (1996). Using language. Cambridge University Press. 
Clark, H. H. \& Brennan, S. E. (1991). Grounding in communication. Perspectives on Socially Shared Cognition, 13(1991), 127-149.

Dickey, D. A. \& Fuller, W. A. (1979). Distribution of the estimators for autoregressive time series with a unit root. Journal of the American Statistical Association, 74(366a), 427431.

Doyle, G. \& Frank, M. C. (2015). Shared common ground influences information density in microblog texts. In Proceedings of the North American Chapter of the Association for Computational Linguistics - Human Language Technologies (naacl-hlt). Denver, DO.

Dwivedi, Y. \& Subba Rao, S. (2011). A test for second-order stationarity of a time series based on the discrete Fourier transform. Journal of Time Series Analysis, 32(1), 6891.

Fusaroli, R., Bahrami, B., Olsen, K., Roepstorff, A., Rees, G., Frith, C., \& Tylén, K. (2012). Coming to terms quantifying the benefits of linguistic coordination. Psychological Science, 23(8), 931-939.

Fusaroli, R., Raczaszek-Leonardi, J., \& Tylén, K. (2014). Dialog as interpersonal synergy. New Ideas in Psychology, 32, 147-157.

Fusaroli, R. \& Tylén, K. (2016). Investigating conversational dynamics: interactive alignment, interpersonal synergy, and collective task performance. Cognitive Science, 40(1), 145-171.

Garrod, S. \& Anderson, A. (1987). Saying what you mean in dialogue: a study in conceptual and semantic co-ordination. Cognition, 27(2), 181-218.

Garrod, S. \& Pickering, M. J. (2009). Joint action, interactive alignment, and dialog. Topics in Cognitive Science, 1(2), 292-304.

Genzel, D. \& Charniak, E. (2002). Entropy rate constancy in text. In Proc. 40th Annual Meeting on Association for Computational Linguistics (pp. 199-206). Philadelphia, PA.

Genzel, D. \& Charniak, E. (2003). Variation of entropy and parse trees of sentences as a function of the sentence number. In Proceedings of the 2003 Conference on Empirical Methods in Natural Language Processing (pp. 65-72). Association for Computational Linguistics.
Godfrey, J. J., Holliman, E. C., \& McDaniel, J. (1992). Switchboard: telephone speech corpus for research and development. In International Conference on Acoustics, Speech, and Signal Processing (Vol. 1, pp. 517520). IEEE. San Francisco, CA.

Gregory Jr, S. W. \& Gallagher, T. J. (2002). Spectral analysis of candidates' nonverbal vocal communication: predicting us presidential election outcomes. Social Psychology Quarterly, 298-308.

Jaeger, T. F. (2010). Redundancy and reduction: speakers manage syntactic information density. Cognitive Psychology, 61(1), 23-62.

Keller, F. (2004). The entropy rate principle as a predictor of processing effort: an evaluation against eye-tracking data. In Proc. conference on Empirical Methods in Natural Language Processing (pp. 317-324). Barcelona, Spain.

Kwiatkowski, D., Phillips, P. C., Schmidt, P., \& Shin, Y. (1992). Testing the null hypothesis of stationarity against the alternative of a unit root: how sure are we that economic time series have a unit root? Journal of Econometrics, 54(1-3), 159-178.

Ljung, G. M. \& Box, G. E. (1978). On a measure of lack of fit in time series models. Biometrika, 297-303.

Narasimhan, S. \& Veena, S. (2005). Signal processing: principles and implementation. Alpha Science Int'l Ltd.

Natrella, M. (2010). Nist/sematech e-handbook of statistical methods. NIST/SEMATECH.

Ng, S. H. \& Bradac, J. J. (1993). Power in language: Verbal communication and social influence. Sage.

Oreström, B. (1983). Turn-taking in english conversation. Lund: CWK Gleerup.

Oullier, O., Bardy, B. G., Stoffregen, T. A., \& Bootsma, R. J. (2002). Postural coordination in looking and tracking tasks. Human Movement Science, 21(2), 147-167.

Oullier, O., De Guzman, G. C., Jantzen, K. J., Lagarde, J., \& Kelso, S. J. (2008). Social coordination dynamics: measuring human bonding. Social Neuroscience, 3(2), 178-192.

Phillips, P. C. \& Perron, P. (1988). Testing for a unit root in time series regression. Biometrika, 335-346. 
Pickering, M. J. \& Garrod, S. (2004). Toward a mechanistic psychology of dialogue. Behavioral and Brain Sciences, 27(02), 169-190.

Pickering, M. J. \& Garrod, S. (2006). Alignment as the basis for successful communication. Research on Language and Computation, 4(2-3), 203-228.

Qian, T. \& Jaeger, T. F. (2011). Topic shift in efficient discourse production. In Proceedings of the 33rd Annual Conference of the Cognitive Science Society (pp. 3313-3318).

Reitter, D. \& Moore, J. D. (2007). Predicting success in dialogue. In Proc. 45th Annual Meeting of the Association of Computational Linguistics (pp. 808-815). Prague, Czech Republic.

Reitter, D. \& Moore, J. D. (2014). Alignment and task success in spoken dialogue. Journal of Memory and Language, 76, 29-46.

Schuller, B., Steidl, S., Batliner, A., Burkhardt, F., Devillers, L., Müller, C., \& Narayanan, S. (2013). Paralinguistics in speech and language-state-of-the-art and the challenge. Computer Speech and Language, 27(1), 4 39.

Shannon, C. E. (1948). A mathematical theory of communication. The Bell System Technical Journal, 27, 379-423.

Stolcke, A. (2002). SRILM - an extensible language modeling toolkit. In The 7th International Conference on Spoken Language Processing. Denver, Colorado.

Xu, Y. \& Reitter, D. (2016a). Convergence of syntactic complexity in conversation. In Proc. 54th Annual Meeting of the Association for Computational Linguistics (Volume 2: Short Papers) (pp. 443-448). Berlin, Germany.

Xu, Y. \& Reitter, D. (2016b, August). Entropy Converges Between Dialogue Participants: Explanations from an InformationTheoretic Perspective. In Proceedings of the 54th Annual Meeting of the Association for Computational Linguistics (Volume 1: Long Papers) (pp. 537-546). Berlin, Germany: Association for Computational Linguistics. 